\title{
Calculation of the fission observables in the resolved resonance energy region of the ${ }^{235} \mathrm{U}(\mathrm{n}, \mathrm{f})$ reaction
}

\author{
Olivier Serot ${ }^{1, *}$, Olivier Litaize ${ }^{1}$, and Abdelhazize Chebboubi ${ }^{1}$ \\ ${ }^{1}$ CEA, DEN, SPRC, LEPh, F-13108 Saint Paul lez Durance, France
}

\begin{abstract}
Measurement of the fission fragments in coincidence with the emitted prompt neutrons was undertaken recently, at JRC-Geel institute, for the ${ }^{235} \mathrm{U}(\mathrm{n}, \mathrm{f})$ reaction in the resolved resonance energy region, up to $160 \mathrm{eV}$ incident neutron energy. From this experimental work, fluctuations of several fission observables (mass yields, average total kinetic energy $\overline{\mathrm{TKE}}$, average prompt neutron multiplicity $\bar{v}_{P}$ ) were clearly observed. In the present work, these experimental pre-neutron fission fragment mass and kinetic energy distributions were used as input data for the FIFRELIN Monte Carlo code. By adopting the Hauser-Feshbach statistical model, the code simulates the de-excitation of the fission fragments. Four free parameters are available in the code: two of them (called $\mathrm{R}_{T}^{\min }$ and $\mathrm{R}_{T}^{\max }$ ) govern at the scission point the sharing of the total available excitation energy between the two nascent fission fragments, while the two others (called $\sigma_{L}$ and $\sigma_{H}$ ) assign the initial fission fragment spins. In this way, fission observables (prompt particles energy spectra and multiplicities, delayed neutrons multiplicity,...) and correlations between them can be predicted and investigated. Here, these four free parameters were tuned in order to reproduce the average prompt neutron multiplicity at the resonance $\mathrm{E}_{n}=19.23 \mathrm{eV}$, resonance for which the experimental statistical uncertainty on $\bar{v}_{P}$ is the lowest one. Then, the calculations were perfomed for all resonances by keeping the same set of free parameters. We show that the calculated fluctuations of $\bar{v}_{P}$ in the resonances can rather be well reproduced by considering only the fluctuations of the pre-neutron mass yields and kinetic energy. In addition, from our calculation procedure, other fission observables fluctuations can also be predicted.
\end{abstract}

\section{Introduction}

In the case of the ${ }^{239} \mathrm{Pu}$ neutron induced fission reaction, fluctuations of the average prompt neutron multiplicity $\bar{v}_{P}$ have been clearly established in the resolved resonance region (see for example [1]). These fluctuations are generally interpreted as the $(\mathrm{n}, \gamma \mathrm{f})$ contribution to the total $(\mathrm{n}, \mathrm{f})$ cross section [2-5]. After the capture of a neutron by the target nucleus, it may happen that the compound nucleus emits a $\gamma$ before undergoing fission. In that case, since the average energy carried away by the pre-fission photon is about $1 \mathrm{MeV}$ [2], the excitation energy available for the fission fragments is lower than in the case of a direct fission, leading to a lower neutron multiplicity. According to this model, the $\bar{v}_{P}$-value is governed by the average $v_{P}^{n, f}$-value associated to the direct $(\mathrm{n}, \mathrm{f})$ contribution and the average $v_{P}^{n, \gamma f}$-value associated to the (n, $\left.\gamma \mathrm{f}\right)$ contribution. Both quantities $\left(v_{P}^{n, f}\right.$ and $\left.v_{P}^{n, \gamma f}\right)$ are assumed to be constant (regardless of the presence of a resonance), only their contributions are fluctuating in the resonances. Since $v_{P}^{n, f}>v_{P}^{n, \gamma f}$, the average prompt neutron multiplicity for a given resonance can only be reduced compared to the resonances where the $(\mathrm{n}, \gamma \mathrm{f})$ contribution is negligible.

The aim of the present work is to investigate similar fluctuations in the resonance region, but in the case of the ${ }^{235} \mathrm{U}(\mathrm{n}, \mathrm{f})$ reaction. As will be seen in Sect. 2, such

*e-mail: olivier.serot@cea.fr fluctuations were experimentally observed not only for the average prompt neutron multiplicity but also for other fission observables (average total kinetic energy of the fission fragments, emission probability of ternary- $\alpha$ particles,...). The recent experiment performed by the JRC-Geel (Belgium) [6-9] is certainly the most accomplished one. Indeed, for the first time, they were able to measure (for 88 resonances) correlations between fission fragments properties (mass and kinetic energy) and prompt neutron multiplicity. By using their fission fragments pre-neutron mass and kinetic energy distributions, we have performed calculations with the Monte Carlo code FIFRELIN (FIssion FRagment Evaporation Leading to an INvestigation of nuclear data) in order to deduce characteristics of the emitted prompt particles (neutrons and $\gamma$ ). These calculations are presented and discussed in Sect. 3.

\section{Comments on Available Experimental Data}

As already said in the Introduction, various experiments have revealed the presence of fission observables fluctuations in the resonance region of the ${ }^{235} \mathrm{U}$ neutron-induced fission reaction. Four types of experiments are reminded here.

- Measurement of the fission fragments properties 


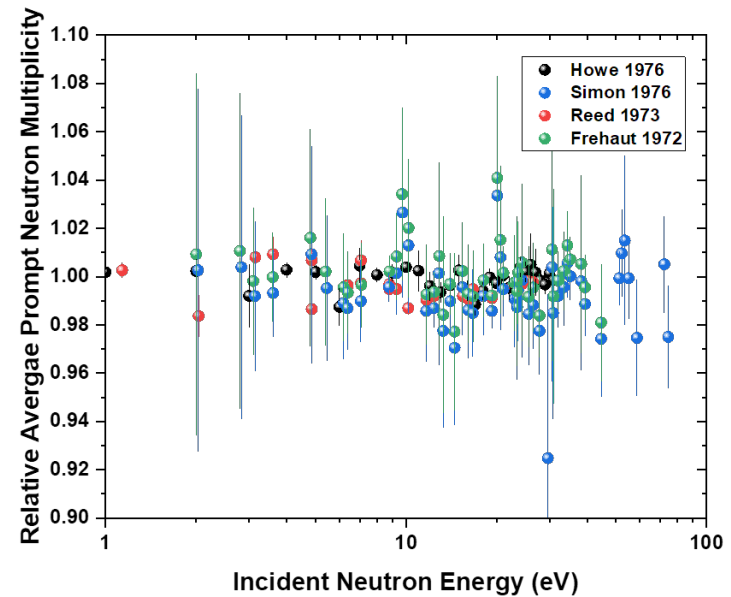

Figure 1. Relative average prompt neutron multiplicity from 1 $\mathrm{eV}$ to $100 \mathrm{eV}$ for the ${ }^{235} \mathrm{U}(\mathrm{n}, \mathrm{f})$ reaction. Data from Howe [12] are relative to the average $v_{P}$-value over incident neutron energies from 0.52 to $114 \mathrm{eV}$. Data from Simon [13] and Reed [14] were renormalized to the thermal $v_{P}$-value and data from Frehaut [15] were renormalized to $v_{P}$-value from ${ }^{252} \mathrm{Cf}(\mathrm{sf})$.

In 1989 , the pre-neutron mass and kinetic energy distributions of the fission fragments were measured by Hambsch et al. [10] for each resonance from $0.006 \mathrm{eV}$ to $130 \mathrm{eV}$. Fluctuations of the average Total Kinetic Energy ( $\overline{\mathrm{TKE}})$ from resonance to resonance were observed with amplitudes up to about $450 \mathrm{keV}$, suggesting possible fluctuations of $\bar{v}_{P}$. No correlation between the spin of the resonance and TKE was found. These experimental results were interpreted by the fluctuations of the socalled Standard I and Standard II fission mode weights (following the Brosa's terminology [11]).

\section{- Measurements of the prompt neutron multiplicity}

Various measurements of the average prompt neutron multiplicity were also carried out in the past, without detection of the fission fragments. Unfortunately, strong discrepancies between those measurements can be observed, preventing a reliable evaluation of the $\bar{v}_{P}$ in the resonances (see Fig. 1).

- Measurements of $\alpha$-emission probability from ternary fission

The ternary $\alpha$-emission probability (usually noted $L R A / B$, where $L R A$ corresponds to the counting rate of the emitted Long Range Alpha particles, and $B$ the total fission events counting rate) was measured by Pommé et al. [17] in the resonance region from thermal up to 100 $\mathrm{eV}$ incident neutron energy. Data were normalized to the average of a few of the strongest resonances. Even if $L R A / B$ shows clear fluctuations, no correlation was established between $L R A / B$ and the spin of the resonance nor with the fission mode weights deduced from the Hambsch's experiment [10].

- Measurement of fission fragments in coincidence with prompt neutrons
The recent experiment performed by Göök et al. [6-9] on the GELINA (GEel LINear Accelerator) facility of the JRC-Geel (Belgium) consisted in measuring fission fragments in coincidence with prompt neutrons. For that, a position-sensitive twin ionization chamber was operated to determine kinetic energy and mass of the fission fragments [16]. In addition, an array of 22 neutron detectors (called SCINTIA) surrounding the ionisation chamber was installed to measure prompt neutrons. Incident neutrons are provided by the GELINA facility and their energy is determined by the Time Of Flight (TOF) technic. With this experimental setup, correlations between fission fragments and prompt neutrons were investigated for 88 resonances from $1 \mathrm{eV}$ up to 160 $\mathrm{eV}$ incident neutron energy. When selecting the 88 resonances, no clear correlation between $\bar{v}_{P}$ and $\overline{\text { TKE }}$ nor between $\bar{v}_{P}$ and $\bar{A}_{H}$ (the average mass of the heavy fission fragment) can be observed (see Figs. 2a and $2 b$ ). Interesting enough is the correlation between $\overline{\mathrm{TKE}}$ and $\bar{A}_{H}$ (see Fig. 2c). This correlation gives confidence in the experimental results, because it suggests that an increase of $\bar{A}_{H}$ leads to a decrease of $\overline{\mathrm{TKE}}$, which is expected by considering the Coulomb potential calculation obtained with the Unchanged Charge Density (UCD) model. Furthermore, by selecting the most accurate Göök's data, namely resonances for which the $\bar{v}_{P}$-value has a statistical uncertainty lower than $1 \%$ (it concerns 12 resonances), correlations expected from physical grounds can be highlighted, as shown in Figs. 2d, 2e and $2 \mathrm{f}$.

Note that Göök et al. have measured the prompt neutron multiplicity relative to the $\bar{v}_{P}$ of ${ }^{252} \mathrm{Cf}$ spontaneous fission, with a detection neutron threshold of $500 \mathrm{keV}$. So, the experimental data $R\left(E_{n}\right)$ provided by Göök for the present work [9], can be written as follows:

$$
R\left(E_{n}\right)=\frac{\bar{v}_{P}^{235 U}\left(E_{n}\right)}{\bar{v}_{P}^{252 C f}} \times \frac{\int_{0.5}^{\infty} \chi^{235 U}\left(E_{n}, E\right) d E}{\int_{0.5}^{\infty} \chi^{252 C f}(E) d E}
$$

where $\chi$ represents the Prompt Fission Neutron Spectrum (PFNS). In order to extract $\bar{v}_{P}^{235 U}\left(E_{n}\right)$ (which are plotted in Fig.2), we assume that the PFNS is independent of the resonance energy: $\int_{0.5}^{\infty} \chi^{235 U}\left(E_{n}, E\right) d E=$ $\int_{0.5}^{\infty} \chi^{235 U}\left(E_{\text {therm }}, E\right) d E$. The ratio of the integrals appearing in Eq.(1) is calculated by considering the ${ }^{235} U\left(n_{\text {therm }}, f\right)$ PFNS given by JEFF-3.3. PFNS for ${ }^{252} C f(s f)$ is also taken from JEFF-3.3. Lastly, we adopt: $\bar{v}_{P}^{252 C f}=3.76$.

From all these experimental results, it becomes clear that the fission observables fluctuations in the resonance region are not of statistical origin, but are based on physical grounds. Moreover, Göök et al. were able to demonstrate for the first time, that the $\bar{v}_{P}$ fluctuations in the resonance are due to the fluctuations of the fission fragment properties.

\section{Calculations performed with the FIFRELIN Monte Carlo code}

The FIFRELIN Monte Carlo code, developped at CEACadarache, aims at calculating post-fission observables 

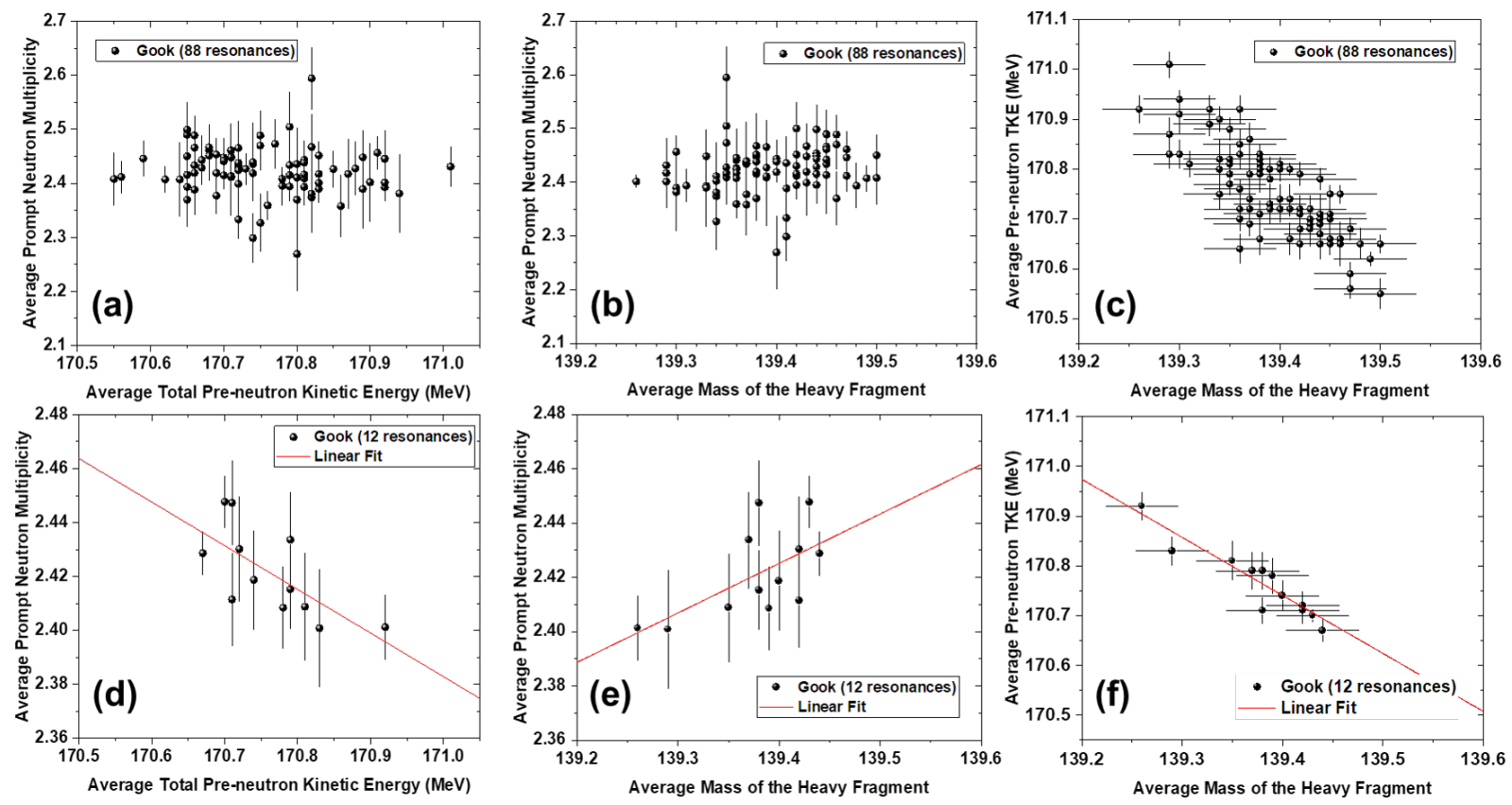

Figure 2. Correlations measured by JRC-Geel $[6,7,9]$ between $\bar{v}_{P}$ and $\overline{\text { TKE }}$ (Fig. (a)), between $\bar{v}_{P}$ and $\bar{A}_{H}$ (Fig. (b)) and between $\overline{\mathrm{TKE}}$ and $\bar{A}_{H}$ (Fig. (c)) for the 88 resonances. The same correlations are plotted in Figs. (d), (e) and (f), but for the 12 most accurate resonances. Red lines correspond to a linear fit performed over experimental points. Note the large differences in scale between subfigures (a), (b) and (c), (d).

(spectra and multiplicities of the prompt neutron and gamma particles; fission energies released). In particular, this code is a nice tool to investigate correlations between fission observables, which can be very useful for improving our understanding of the fission process. All the details related to this code can be found in Refs. [18-20].

\subsection{Procedure used for the calculations}

We use FIFRELIN for the 88 resonances measured by Göök and co-workers. For each resonance, the calculation procedure is done following several successive steps which are briefly described here.

- Mass. The first step consists in the determination of the light fragment mass $A_{L}$ (before prompt neutron emission) which is done by sampling the measured preneutron mass distribution. The mass $A_{H}$ of the heavy partner is deduced from the mass conservation law.

- Kinetic energy. For a given mass, the kinetic energy is assumed to be a Gaussian type distribution, where the average value $\overline{\mathrm{KE}}(A)$ and the width $\sigma_{K E}(A)$ are given by the experiment. By sampling a kinetic energy value $\mathrm{KE}\left(A_{L}\right)$ for the mass $A_{L}$ (selected in the previous step), the kinetic energy for the complementary mass $\operatorname{KE}\left(A_{H}\right)$ is deduced from the linear momentum conservation law.

- Nuclear Charge. The nuclear charges $\left(Z_{L}\right.$ and $\left.Z_{H}\right)$ of both fission fragments are calculated from the Wahl systematic [21] which takes into account the charge polarisation and the odd-even effects.
- Excitation energy. The Total Excitation Energy (TXE) available at scission can then be deduced from energy conservation: $T X E=Q+E_{n}+B_{n}-T K E=$ $B\left(A_{L}, Z_{L}\right)+B\left(A_{H}, Z_{H}\right)-B\left(A_{f}, Z_{f}\right)+E_{n}+B_{n}-T K E$, where $B\left(A_{L}, Z_{L}\right), B\left(A_{H}, Z_{H}\right)$ and $B\left(A_{f}, Z_{f}\right)$ are the binding energies of the light fragment, the heavy fragment and the fissioning nucleus, respectively. $E_{n}$ is the incident neutron energy and $B_{n}$ the neutron separation energy of the compound nucleus $\left({ }^{236} U\right)$. The partitioning of TXE between the light and the heavy fragments is done from an empirical mass dependent temperature ratio $R_{T}=T_{L} / T_{H} \equiv R_{T}(A)$, where $T_{L}$ and $T_{H}$ are the nuclear temperature of the light and heavy fragments after their full acceleration and after recovering their ground state deformation. The $R_{T}$-function is defined from physical constraints based on the expected nucleus deformation at scission (see Ref.[18] for the details). Two free parameters $\left(R_{T}^{\min }\right.$ and $\left.R_{T}^{\max }\right)$ are needed in order to define the $R_{T}$-values for all fission fragment masses.

- Spin $J$ and parity $\pi$. The spin $J$ of the fission fragments are sampled from a Rayleigh type distribution:

$$
\left.P(J) \approx \frac{(2 J+1)}{2 \sigma^{2}} \exp \left(-(J+1 / 2)^{2}\right) / 2 \sigma^{2}\right)
$$

where $\sigma$ is the spin-cutoff parameter. In the present work, $\sigma$ is assumed to be independent of the excitation energy. Instead, a constant value $\sigma_{L}\left(\sigma_{H}\right)$ is chosen to characterize the spin distribution of all light (heavy) fission fragments. $\sigma_{L}$ and $\sigma_{H}$ are two additional free parameters. The parity $\pi$ is assumed to be positive or negative with a probability: $P(\pi=+)=P(\pi=-)=0.5$. 

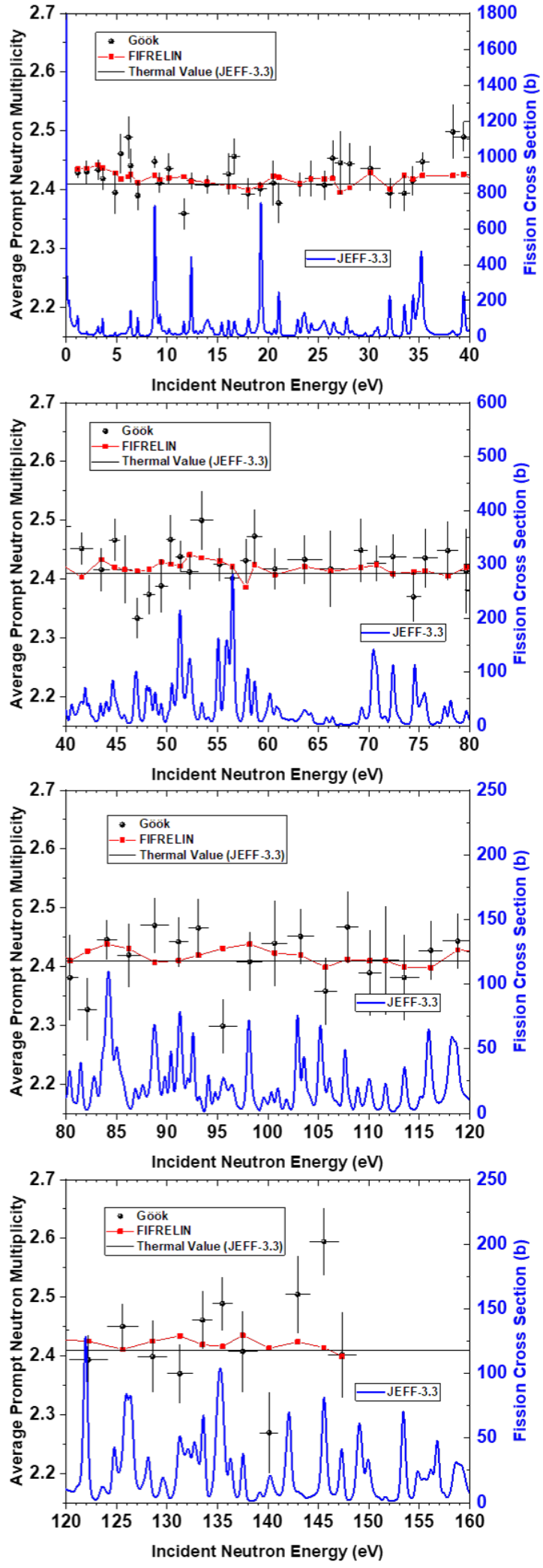

Figure 3. Average prompt neutron multiplicity for the 88 resonances measured by Göök [9]. For each experimental point (black dots), the horizontal line indicates the energy range considered by the experimentalist while the vertical line corresponds to the statistical uncertainty. The black line gives the thermal $v_{P^{-}}$ value from JEFF-3.3. The red points were obtained from FIFRELIN calculation. Lastly, the fission cross-section given by JEFF3.3 is plotted (blue line) and must be read on the right scale.

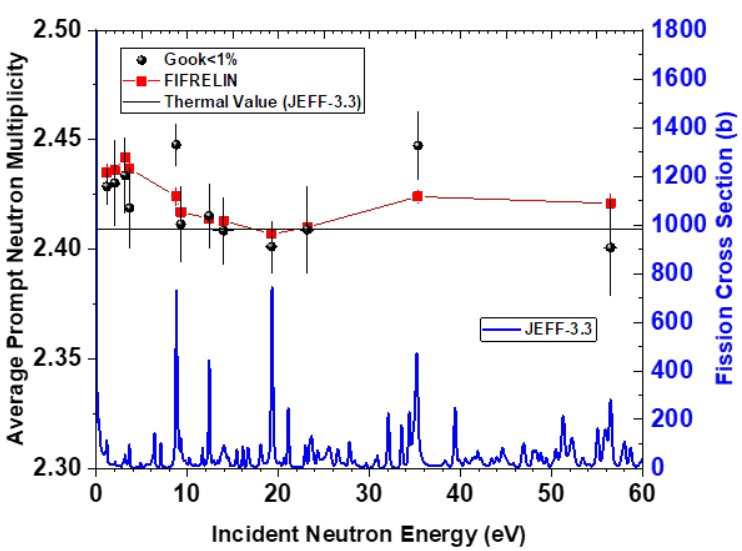

Figure 4. Same as in Fig. 3, but for the 12 more accurate resonances (statistical uncertainties lower than $1 \%$ ).

- De-excitation of the fission fragments. Once all previous steps are completed, both fission fragments are fully characterized. Then, the code simulates the deexcitation of the FF by using the Hauser-Feshbach formalism [22] and the procedure proposed by Becvar [23]. In this way, the competition between prompt neutron and prompt gamma emission is properly taken into account. The conservation laws for the energy, spin and parity of the initial and final states are also respected. All the details related to the numerical procedure used for this de-excitation step are described in Ref. [20].

In the present work, the four free parameters $\left(\sigma_{L}, \sigma_{H}, R_{T}^{\min }\right.$ and $R_{T}^{\max }$ ) are adjusted to reproduce the average prompt neutron multiplicity at the resonance $E_{n}=19.23 \mathrm{eV}: \bar{v}_{P}=$ $2.401 \pm 0.012$. The choice of this resonance originates from the fact that the statistical uncertainty is the lowest one $(0.5 \%)$ among the 88 measured resonances. We found: $R_{T}^{\min }=0.87, R_{T}^{\max }=1.20, \sigma_{L}=7.75 \hbar$ and $\sigma_{H}=8.25 \hbar$. Then, the same parameters are kept for all resonances. In this way, the impact of the pre-neutron mass yields and kinetic energy measured by Göök on $\bar{v}_{P}$ can be investigated. For each resonance, 200000 fission events were simulated. With this number of events, the statistical uncertainties on most of the calculated fission observables is of the order of $0.2 \%$.

\subsection{Comparison with Göök’s experimental data}

Fig. 3 shows the experimental average prompt neutron multiplicity from Göök [9] compared to the FIFRELIN calculations for the 88 resonances between $1 \mathrm{eV}$ and 160 eV. It can be observed that FIFRELIN calculations reproduce rather well the experimental data, except for five resonances $\left(\mathrm{E}_{n}=11.7 \mathrm{eV}, 39.4 \mathrm{eV}, 47.1 \mathrm{eV}, 95.5 \mathrm{eV}\right.$ and 145.6 $\mathrm{eV})$, where the discrepancy is larger than two standard deviations. When considering only the 12 most accurate resonances, the agreement becomes excellent (see Fig. 4). This result is important because it demonstrates that the fluctuations of the fission fragment properties (mass and 


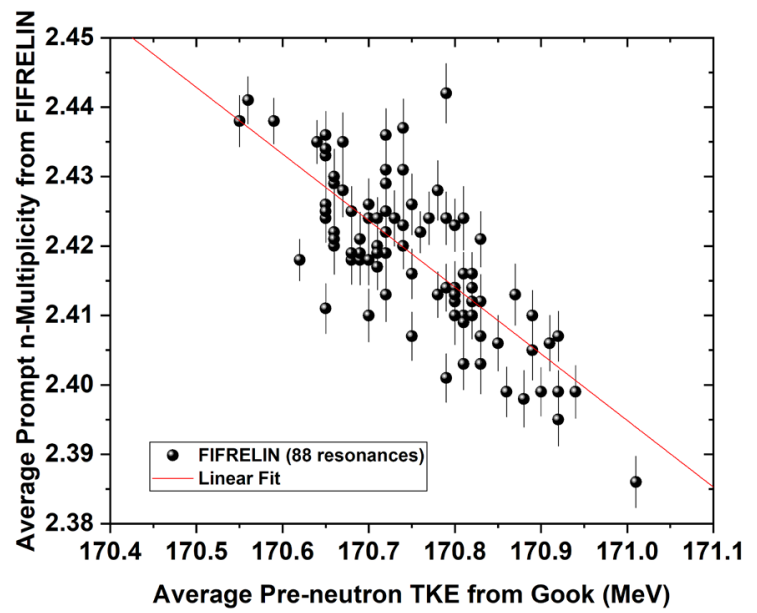

Figure 5. Average prompt neutron multiplicity calculated with FIFRELIN as a function of the average fission fragment total kinetic energy measured by Göök, for 88 resonances.

kinetic energy) measured by Göök and used by FIFRELIN allow to nicely reproduce the $\bar{v}_{P}$ fluctuations.

Fig. 5 shows the $\bar{v}_{P}$-values calculated by FIFRELIN as a function of the average fission fragment pre-neutron total kinetic energy measured by Göök, for the 88 resonances. By making a linear fit through the data, we learn that by increasing $\overline{\mathrm{TKE}}$ by $400 \mathrm{keV}$, we decrease $\bar{v}_{P}$ by 0.04 neutrons. This is in agreeement with the results shown in Fig. 2d. According to this figure, $\bar{v}_{P}$ is expected to fluctuate around the thermal value (2.41, according to JEFF3.3).

\subsection{Prediction of other fission observables}

According to the FIFRELIN calculations, the $\bar{v}_{P}$ fluctuations are accompanied by fluctuations of the prompt gamma multiplicity $\bar{M}_{\gamma}$, as shown in Fig. 6a. Here, $\bar{M}_{\gamma}$ is calculated for $[0-10 \mathrm{MeV}] \gamma$-energy range (i.e. without $\gamma$ energy threshold). By averaging the prompt gamma multiplicity over all resonances, a value of $7.60 \gamma / \mathrm{f}$ was found. In addition, a slight positive correlation (see Fig. 6b) between $\bar{M}_{\gamma}$ and $\bar{v}_{P}$ was observed.

Concerning the average energy (in Laboratory System) of the PFNS (see Fig. 7), the low fluctuations seem to be from statistical origin. No correlation was observed between the average total available excitation energy and the average energy of the PFNS. The average value obtained here $\left(\bar{E}_{P F N S}=1.934 \mathrm{MeV}\right)$ is slightly lower than the value measured by Kornilov [24] $\left(\bar{E}_{P F N S}=1.988 \mathrm{MeV}\right)$ for the thermal neutron induced fission of ${ }^{235} \mathrm{U}$.

\section{Conclusion and perspectives}

The recent experiment performed at JRC-Geel by Göök and co-workers, has revealed, for the first time, clear correlations between the fission fragments characteristics (mass
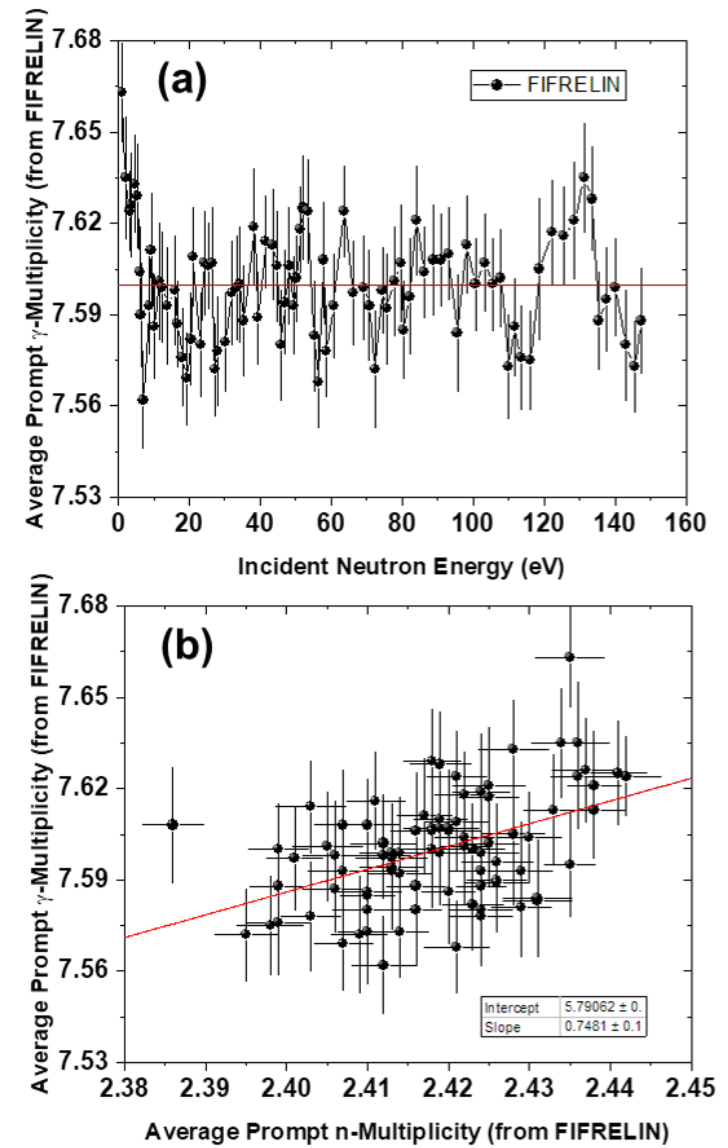

Figure 6. (a): Average prompt gamma multiplicity calculated with FIFRELIN for the 88 resonances. (b) Correlation between the average prompt gamma multiplicity and the prompt neutron multiplicity for the same 88 resonances.

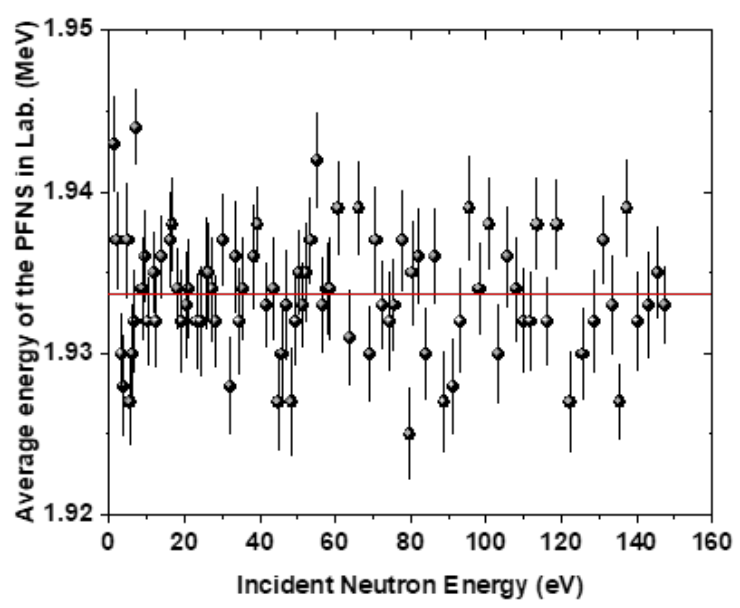

Figure 7. Average energy of the prompt neutron energy spectra calculated with FIFRELIN for the 88 resonances, as a function of the incident neutron energy. 
and kinetic energy) and the average prompt neutron multiplicity for 88 resonances from $1 \mathrm{eV}$ to $160 \mathrm{eV}$ incident neutron energy. In particular, when looking at the 12 resonances for which the statistical uncertainty on $\bar{v}_{P}$ is lower than $1 \%$, those correlations appear to be based on physical grounds (see Fig. 2a, 2b and 2c).

By using the pre-neutron fission fragment mass and kinetic energy distributions measured by Göök, we have performed calculations of various fission observables (multiplicity and spectra of the emitted prompt neutrons and gamma) by using the FIFRELIN Monte Carlo code. From these calculations, we were able to reproduce experimental data on $\bar{v}_{P}$ (see Fig. 3 and 4), but also to predict fluctuations of other fission observables.

This work shows that fluctuations of $\bar{v}_{P}$ in the resonances are more due to the fluctuations of the fission fragments properties than to the $(\mathrm{n}, \gamma \mathrm{f})$ process. Even if this latter effect is not excluded, it is expected to be low, since according to Ref. [2], the $\Gamma_{\gamma f}$ widths for the 3- and 4resonances were assessed to $2.3 \mathrm{meV}$ and $1.4 \mathrm{meV}$, respectively. Thanks to these $\Gamma_{\gamma f}$-values, the $(\mathrm{n}, \gamma \mathrm{f})$ process is probably lower for the ${ }^{235} \mathrm{U}(\mathrm{n}, \mathrm{f})$ reaction than for the ${ }^{239} \mathrm{Pu}(\mathrm{n}, \mathrm{f})$.

It is interesting to note that in the evaluated nuclear data libraries, the description of $\bar{v}_{P}$ in the resonances show strong discrepancies between each other. For instance, fluctuations of $\bar{v}_{P}$ were introduced in JEF-2.2, but disappeared in the most recent version JEFF-3.3. We believe that these fluctuations might be introduced again by following the procedure described in the present work.

\section{Acknowledgements}

We would like to acknowledge A. Göök and F.-J. Hambsch for providing us their experimental data and for their stimulating and fruitfull discussions.

\section{References}

[1] J. Fréhaut, and D. Shackleton, Proc. 3rd Symp. Physics and Chemistry of Fission, Rochester, New York, Aug. 13-17 (1973).
[2] J.E. Lynn, P. Talou, and O. Bouland, Phys. Rev. C 97, 064601 (2018).

[3] E. Fort et al., Nucl. ScI. ENG. 99, 375-389 (1988).

[4] G. Noguere, O. Serot, WPEC-34, NEA/NSC/WPEC Doc 447 (2014).

[5] E. Leal-Cidoncha, G. Noguere, O. Bouland, and O. Serot, EPJ Web of Conferences 211, 02004 (2019).

[6] F.-J. Hambsch, P. Salvador-Castiñeira, S. Oberstedt, A. Göök, and R. Billnert, EPJ Web of Conferences 122, 01005 (2016).

[7] A. Göök, F.-J. Hambsch, and S. Oberstedt, EPJ Weв of Conferences 146, 04007 (2017).

[8] A. Göök, F.-J. Hambsch, S. Oberstedt, and M. Vidali, Phys. Rev. C 98, 044615 (2018).

[9] A. Göök, private communication (2019).

[10] F.-J. Hambsch, H.-H. Knitter, C. Budtz-Jørgensen, J.P. Theobald, Nucl. Phys. A 491, 56 (1989).

[11] U. Brosa, S. Grossmann, and A. Muller, Phys. ReP 197, 167 (1990).

[12] R.E. Howe, T.W. Phillips, and C.D. Bowman, Phys. Rev. C 13, 195 (1976).

[13] T. Simon, Ph.-D. thesis, 1976.

[14] R.L. Reed, Ph.-D. thesis, Rensselaer Polytechnic Institute, Troy, New York (1973).

[15] Fréhaut et al., Phys. Lett. B 42, 344 (1972).

[16] A. Göök, W. Geerts, F.-J. Hambsch, S. Oberstedt, M. Vidali, and Sh. Zeynalov, Nuclear Instruments AND Methods in Physics Research A 830, 366-374 (2016).

[17] S. Pommé, C. Wagemans, Nucl. Phys. A 587, 1 (1995).

[18] O. Litaize, O. Serot, Phys. Rev. C 82, 054616 (2010).

[19] O. Litaize, O. Serot, L. Berge, Eur. Phys. J. A. 51, 177 (2015).

[20] D. Regnier, O. Litaize, O. Serot, Comp. Phys. CoмMUN. 201, 19 (2016).

[21] A.C. Wahl, LANL Report LA-13928 (2002).

[22] W. Hauser, and H. Feshbach, Phys. Rev. 87, 366 (1952).

[23] F. Becvar, Nucl. Instrum. Meth. Phys. Res. A 417, 434 (1998).

[24] N. Kornilov et al., Nucl. ScI. ANd ENG. 165, 117-127 (2010). 